\title{
Modeling of Neck Effect in Cylindrical Shell
}

\author{
Adam Piwowarczyk ${ }^{1, a}$, Artur Ganczarski ${ }^{2, b^{*}}$ \\ ${ }^{1}$ Institute of Applied Informatics, Cracow University of Technology, Al. Jana Pawła II 37, 30-860 \\ Kraków, Poland \\ ${ }^{2}$ Institute of Applied Mechanics, Cracow University of Technology, Al. Jana Pawła II 37, 30-860 \\ Kraków, Poland \\ aadam.piwowarczyk@mech.pk.edu.pl, bartur.ganczarski@pk.edu.pl
}

Keywords: Neck Effect, Cylindrical Shell, Low Cycle Fatigue

\begin{abstract}
This work presents two models of a neck effect appearing in a cylindrical shell. In the first case, an elastic deformation is described by the conventional shell equation in which axial force appears as an external loading. In the second case, an elastic-plastic deformation including damage effect is described by the kinetic theory of damage evolution implemented to FEM code. Results obtained from both models confirm existence of a distinct displacement, stress and damage localization in the cylindrical shell free from any geometrical or material imperfections.
\end{abstract}

\section{Introduction}

A final stage of the uniaxial tension test is accompanied by characteristic local contraction of a specimen called neck effect. Aforementioned phenomenon is well visible in elongation-force or strain-nominal stress diagram since it corresponds to the beginning of unstable deformation, usually associated with reaching of the ultimate strength. During the necking prior to fracture, originally uniaxial and homogeneous stress state in a specimen becomes inhomogeneous and triaxial. In case of circular specimen analysis of 3D stress state was originally presented by Bridgman [1] and independently by Davidenkov and Spiridonova [3]. In early sixties Cowper and Onat published the paper [2] on neck effect accompanying plane state of strain. In case of a thin-walled tubular specimen the stress state was analyzed by Malinin and Rżysko [6] by use of Laplace's equation. Original numerical analysis of necking process in a bar made of material subjected to rigid-plastic hardening was presented by Dietrich [11]. The assumption of specific format of local displacement rate field was used next to determine a slip system and a range of plastic zone preceding the neck formation.

Generally, the neck problem is still treated as complicated and not sufficiently recognized. The main reason is inhomogeneity of plastic properties, generally exhibiting random distribution, which causes that location of neck is not known in advance. As a consequence, in majority of approaches the neck is treated as a notch of a priori known profile and location.

\section{General formulation of cylindrical shell}

Elastic range

A thin-walled cylindrical shell of variable thickness is considered as a prototype of the tubular specimen made of aluminum alloy Al-2024 shown in Fig. 1. 


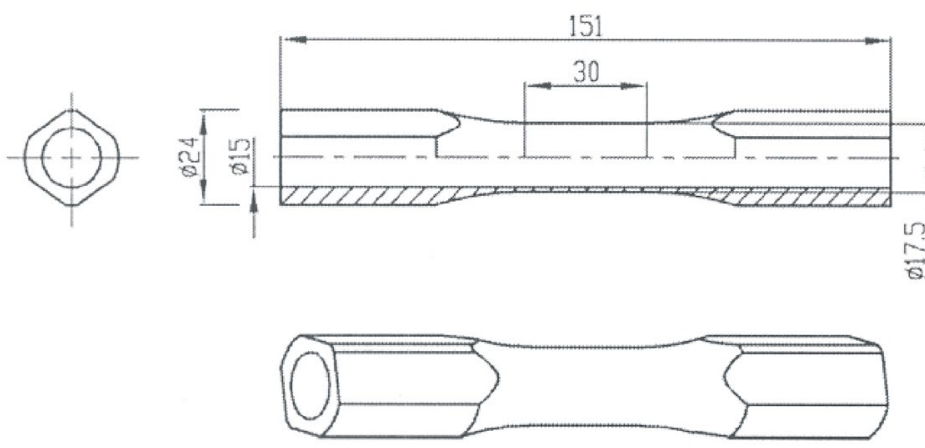

Fig. 1. Geometry of tubular specimen made of aluminum alloy Al-2024.

Conventional differential equation of the cylindrical shell subjected to axial force is as follows

$$
\frac{\mathrm{d}^{2}}{\mathrm{~d} x^{2}}\left[\frac{E h^{3}(x)}{12\left(1-v^{2}\right)} \frac{\mathrm{d}^{2} w}{\mathrm{~d} x^{2}}\right]+\frac{E h(x)}{R^{2}} w=-\frac{v n}{R},
$$

where the right hand side is of special format. Namely, although the problem under consideration deals with the shell loaded by axial force $n$ exclusively, Poisson's effect $-v n / R$ formally corresponds to external pressure. In case of clamped edge of shell, what refers to the tubular specimen having shoulders filled in by pivots, aforementioned term is directly responsible for displacement localization.

Elastic-plastic range including damage

Elastic-plastic range including damage effect of the cylindrical shell is described by use of kinetic theory of damage evolution invented by Lemaitre and Chaboche [5]. Key point of this formalism is dissipation potential

$$
F=\left[\frac{3}{2}\left(\widetilde{\boldsymbol{s}}-\boldsymbol{X}^{\prime}\right):\left(\widetilde{\boldsymbol{s}}-\boldsymbol{X}^{\prime}\right)\right]^{1 / 2}-R-\sigma_{\mathrm{y}}+\frac{3}{4 X_{\infty}} \boldsymbol{X}^{\prime}: \boldsymbol{X}^{\prime}+\frac{Y^{2}}{2 S(1-D h)} \mathrm{H}\left(p-p^{\mathrm{D}}\right),
$$

where $\tilde{\boldsymbol{s}}$ stands for the deviator of effective stress, which takes format

$$
\tilde{\boldsymbol{S}}=\frac{\boldsymbol{\sigma}-\frac{1}{3} \operatorname{Tr}(\boldsymbol{\sigma}) \mathbf{1}}{1-D h}
$$

The last part of the dissipation potential, called the damage potential, is a function of cumulative plastic strain $p$ activated, when damage threshold $p^{\mathrm{D}}$ is attained. The thermodynamic force conjugated to damage $Y$ is equal to the amount of the elastic energy density

$$
Y=\frac{1}{2} \boldsymbol{E}^{-1}: \widetilde{\boldsymbol{\sigma}}: \widetilde{\boldsymbol{\sigma}}
$$

Then applying the formalism of associated plasticity one can find increments of following variables: plastic strain $\mathrm{d} \boldsymbol{\varepsilon}^{\mathrm{p}}$, cumulative plastic strain $\mathrm{d} p$, strain conjugated to isotropic $\mathrm{d} r$ and kinematic hardening $\mathrm{d} \boldsymbol{\alpha}$, damage $\mathrm{d} D$ 


$$
\begin{array}{cc}
\mathrm{d} \boldsymbol{\varepsilon}^{\mathrm{p}}=\frac{\partial F}{\partial \boldsymbol{\sigma}} \mathrm{d} \lambda=\frac{3}{2} \frac{\tilde{\boldsymbol{s}}-\boldsymbol{X}^{\prime}}{\left[\frac{3}{2}\left(\tilde{\boldsymbol{s}}-\boldsymbol{X}^{\prime}\right):\left(\tilde{\boldsymbol{s}}-\boldsymbol{X}^{\prime}\right)\right]^{1 / 2}} \frac{\mathrm{d} \lambda}{1-D h}, \quad \mathrm{~d} p=\left(\frac{2}{3} \mathrm{~d} \boldsymbol{\varepsilon}^{\mathrm{p}}: \mathrm{d} \boldsymbol{\varepsilon}^{\mathrm{p}}\right)^{1 / 2}=\frac{\mathrm{d} \lambda}{1-D h}, \\
\mathrm{~d} r=-\frac{\partial F}{\partial R} \mathrm{~d} \lambda=(1-D h) \mathrm{d} p, \quad \mathrm{~d} \boldsymbol{\alpha}=-\frac{\partial F}{\partial X^{\prime}} \mathrm{d} \lambda=\mathrm{d} \boldsymbol{\varepsilon}^{\mathrm{p}}(1-D h)-\frac{3}{2 X_{\infty}} \boldsymbol{X}^{\prime} \mathrm{d} \lambda, \\
\mathrm{d} D=\frac{\partial F}{\partial Y} \mathrm{~d} \lambda=\frac{Y}{S} \mathrm{H}\left(p-p^{\mathrm{D}}\right) \mathrm{d} p .
\end{array}
$$

In the next step, the inner variables $r, \boldsymbol{\alpha}$, and $Y$ appearing in (5) are expressed by subsequent thermodynamic forces $R, \boldsymbol{X}$ and $D$, calculated form the potential of free energy

$$
\rho \psi=\frac{1}{2} \boldsymbol{E}: \boldsymbol{\varepsilon}^{\mathrm{e}}: \boldsymbol{\varepsilon}^{\mathrm{e}}(1-D h)+R_{\infty}\left[r+\frac{1}{b} \exp (-b r)\right]+\frac{\gamma X_{\infty}}{3} \boldsymbol{\alpha}: \boldsymbol{\alpha} .
$$

Namely, taking appropriate derivatives of (6) with respect to $\boldsymbol{\varepsilon}^{\mathbf{e}}, \boldsymbol{r}$ and $\boldsymbol{\alpha}$, it is possible to convert kinematic inner variables of equations (5) to their stress like equivalents

$$
\begin{array}{cc}
\boldsymbol{\sigma}=\rho \frac{\partial \psi}{\partial \boldsymbol{\varepsilon}}=\boldsymbol{E}: \boldsymbol{\varepsilon}^{\mathrm{e}}(1-D h), & R=\rho \frac{\partial \psi}{\partial r}=R_{\infty}[1-\exp (-b r)], \\
\boldsymbol{X}=\rho \frac{\partial \psi}{\partial \boldsymbol{\alpha}}=\frac{2}{3} \gamma X_{\infty} \boldsymbol{\alpha}, & Y=-\rho \frac{\partial \psi}{\partial D}=\frac{1}{2} \boldsymbol{E}: \boldsymbol{\varepsilon}^{\mathrm{e}}: \boldsymbol{\varepsilon}^{\mathrm{e}} h
\end{array}
$$

achieving final format of constitutive equations for plastic range

$$
\begin{array}{cc}
\boldsymbol{\sigma}=\boldsymbol{E}:\left(\boldsymbol{\varepsilon}-\boldsymbol{\varepsilon}^{\mathrm{p}}\right)(1-D h), & \mathrm{d} R=b\left(R_{\infty}-R\right) \mathrm{d} \lambda, \\
\mathrm{d} \boldsymbol{X}^{\prime}=\gamma\left[\frac{2}{3} X_{\infty} \mathrm{d} \boldsymbol{\varepsilon}^{\mathrm{e}}(1-D h)-\boldsymbol{X}^{\prime} \mathrm{d} \lambda\right], & \mathrm{d} D=\frac{\boldsymbol{E}^{-1}: \boldsymbol{\sigma}: \boldsymbol{\sigma}}{2 S(1-D h)^{2}} \mathrm{H}\left(p-p^{\mathrm{D}}\right) \mathrm{d} p
\end{array}
$$

The magnitude of the plastic multiplier $\mathrm{d} \lambda$ is calculated from the compatibility condition

$$
\frac{\partial F}{\partial \boldsymbol{s}}: \mathrm{d} \boldsymbol{s}+\frac{\partial F}{\partial \boldsymbol{X}^{\prime}}: \mathrm{d} \boldsymbol{X}^{\prime}+\frac{\partial F}{\partial R}: \mathrm{d} R=0,
$$

and it is equal to

$$
\mathrm{d} \lambda=\frac{\frac{3}{2}\left(\tilde{\boldsymbol{s}}-\boldsymbol{X}^{\prime}\right) \mathrm{d} \boldsymbol{\sigma}^{\prime}}{(1-D h)\left[\frac{3}{2}\left(\tilde{\boldsymbol{s}}-\boldsymbol{X}^{\prime}\right):\left(\tilde{\boldsymbol{s}}-\boldsymbol{X}^{\prime}\right)\right]^{1 / 2}\left[\gamma X_{\infty}+b\left(R_{\infty}-R\right)-\frac{3}{2}\left(\tilde{\boldsymbol{s}}-\boldsymbol{X}^{\prime}\right)\left(\frac{\tilde{\boldsymbol{s}}}{1-D h} \frac{\partial F}{\partial Y}+\gamma \boldsymbol{X}^{\prime}\right)\right]} .
$$

The damage deactivation parameter $h$ is defined by formula

$$
h(\sigma)=h_{c}+\left(1-h_{c}\right) \frac{\chi(\sigma)-\chi\left(\sigma_{\mathrm{e}}\right)}{\chi\left(\sigma_{\mathrm{b}}\right)-\chi\left(\sigma_{\mathrm{e}}\right)}
$$

where Hayhurst's function $\chi(\boldsymbol{\sigma})$ is used to combine uniaxial damage $D$ and tridimensional state of stress $\boldsymbol{\sigma}$ (see Cegielski [8], Ganczarski and Cegielski [9]).

\section{Results}

In case of the cylindrical shell working in elastic range, the fourth order differential equation (1) is numerically integrated from $x_{1}=0 \mathrm{~mm}$ to $x_{2}=75 \mathrm{~mm}$ by a use of the finite difference routine as well as the fourth-order Runge-Kutta technique (see Press et al. [10]), which require application of following boundary conditions

$$
w\left(x_{1}\right)=0, \quad w^{\prime}\left(x_{1}\right)=\left.\frac{\mathrm{d} w}{\mathrm{~d} x}\right|_{x_{1}}=0, \quad w^{\prime \prime}\left(x_{2}\right)=\left.\frac{\mathrm{d}^{2} w}{\mathrm{~d} x^{2}}\right|_{x_{2}}=0, \quad w^{\prime \prime \prime}\left(x_{2}\right)=\left.\frac{\mathrm{d}^{3} w}{\mathrm{~d} x^{3}}\right|_{x_{2}}=0 .
$$


Distributions of displacement function $w(x)$ and its second differential $w^{\prime \prime}(x)$, directly corresponding to the bending moment $m_{x}(x)=\frac{E h^{3}(x)}{12\left(1-v^{2}\right)} w^{\prime \prime}(x)$, exhibit local extrema located in relatively narrow range $x \in(55,60)$, referring to the gauge length adjacent to transition region of conical profile (see Fig. 2).

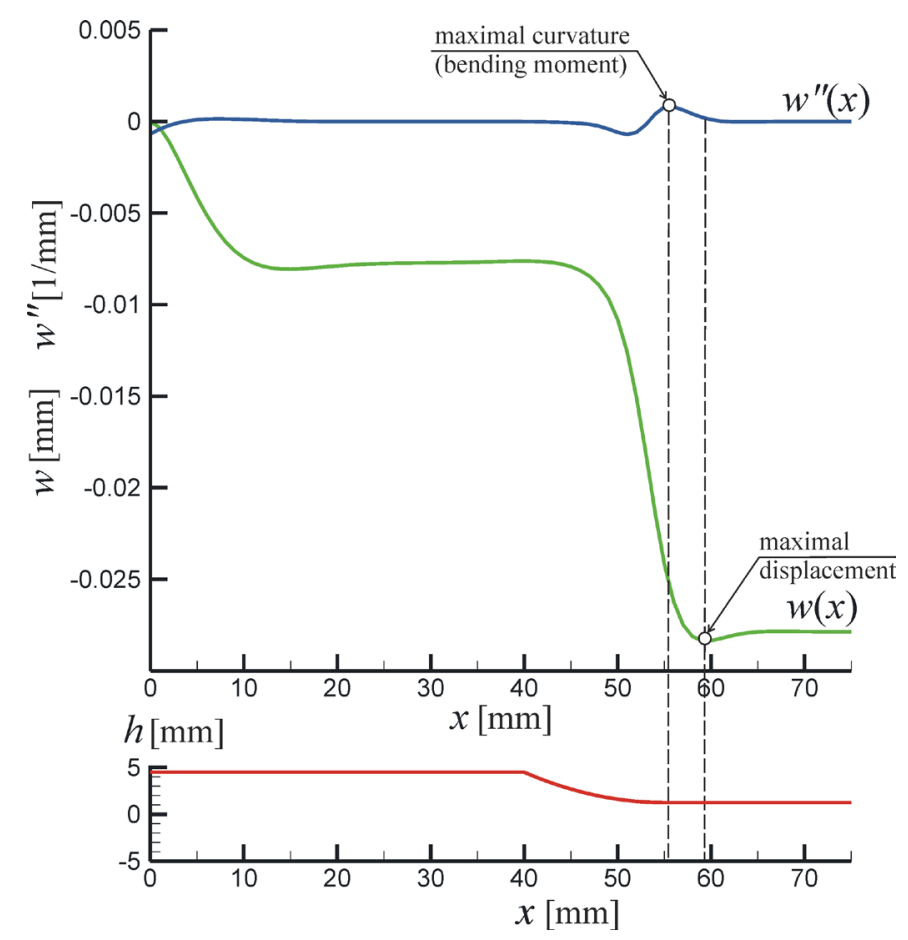

Fig. 2. Distributions of displacement function $w(x)$ and its second differential $w^{\prime \prime}(x)$ compared to profile of cylindrical shell.

In case of the cylindrical shell working in elastic-plastic range, system of equations (8-11) is implemented to the Finite Element Method (FEM) code for quadrilateral axisymmetric element (see Owen and Hinton [7], Ganczarski and Skrzypek [4]) .

Complete material data of the aluminum alloy Al-2024 is presented in Tab. 1.

Tab. 1. Material data of the aluminum alloy Al-2024 (see Ganczarski and Cegielski [9]).

\begin{tabular}{|c|c|c|c|c|c|c|c|c|c|}
\hline$E[\mathrm{GPa}]$ & $\sigma_{\mathrm{y}}[\mathrm{MPa}]$ & $b$ & $\gamma$ & $v$ & $p^{D}$ & $S[\mathrm{GPa}]$ & $R_{\infty}[\mathrm{MPa}]$ & $X[\mathrm{MPa}]$ & $\rho\left[\mathrm{kg} / \mathrm{m}^{3}\right]$ \\
\hline 70 & 230 & 0.1 & 4.0 & 0.3 & 0.248 & 3.5 & 120 & 60 & $2.7 \cdot 10^{6}$ \\
\hline
\end{tabular}

Two types of displacement-control low cycle fatigue processes are analyzed: a pendulum like tension-compression $\Delta l= \pm 0.75 \mathrm{~cm}$ and a pulsating tension $0 \leq \Delta l \leq 0.75 \mathrm{~cm}$. Similarly to the elastic range, first nucleation of plasticity and corresponding damage takes place in the gauge length adjacent to transition region of conical profile. However, combined effects of isotropic and kinematic plastic hardening cause motion of the damage front, which finally localizes in the middle of gauge length (see Fig. 3). 

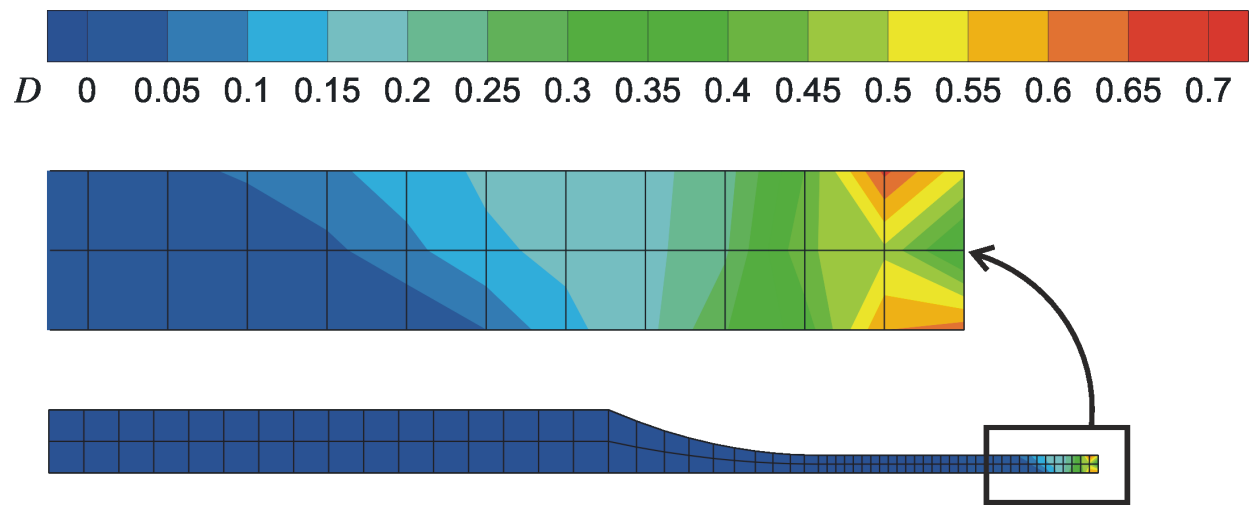

Fig. 3. Distribution of damage $D$ in the whole mesh and localization region.

Displacement field corresponding to the initiation of first micro-crack exhibits clear localization (see Fig. 4).

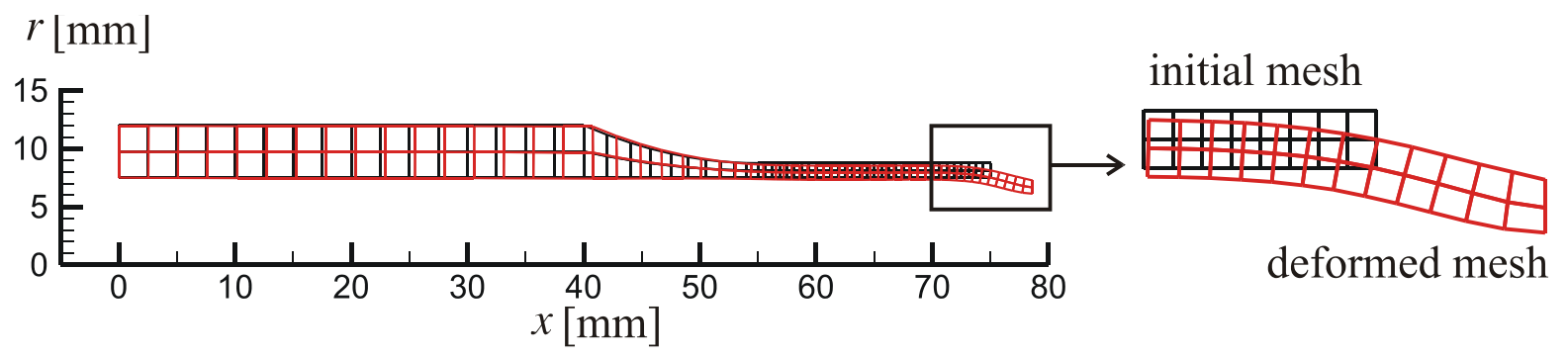

Fig. 4. Mesh: initial (black) and deformed (red) with localization region.

In case of the pendulum-like tension-compression cycle (see Fig. 5a) displacement-force diagram performs regular hysteresis loops, which exhibit stronger effect of a force amplitude decrease

a)

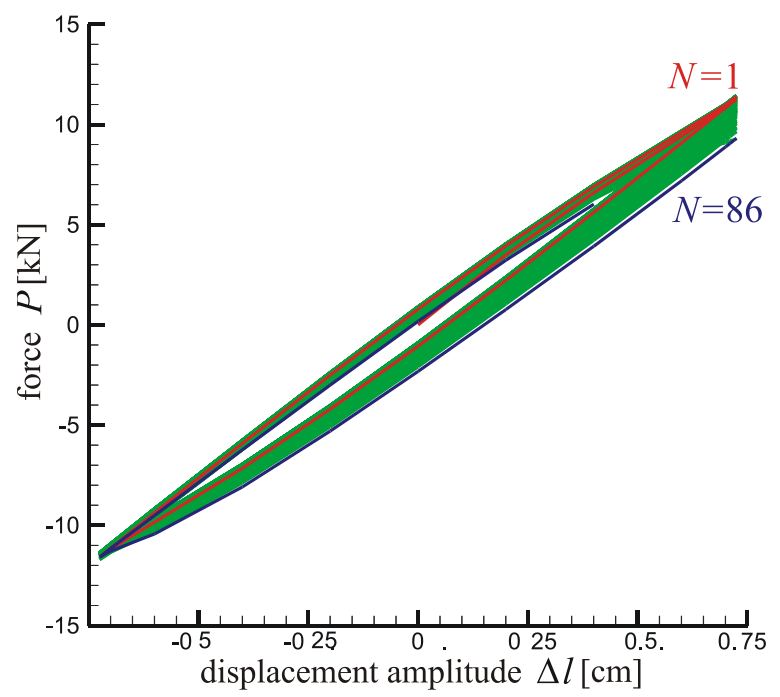

b)

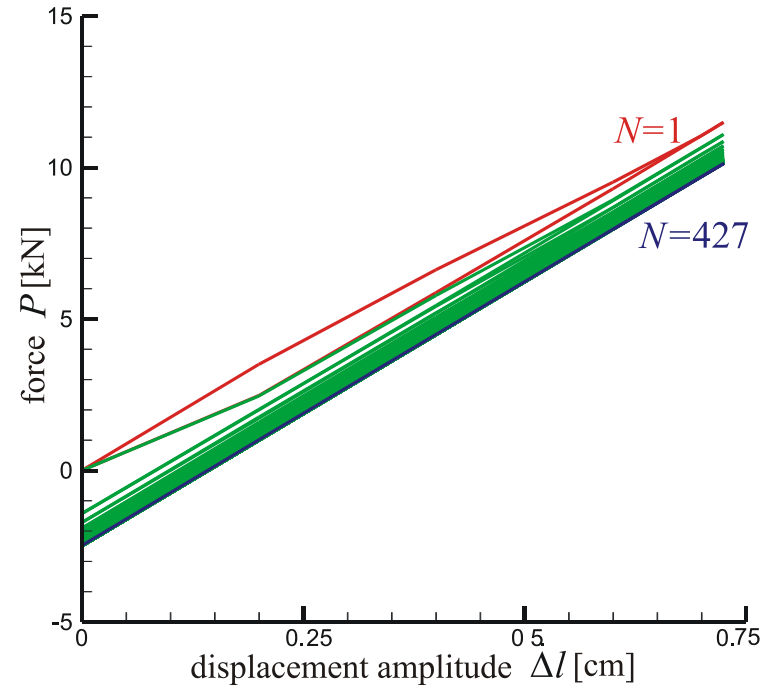

Fig. 5. Hysteresis loops corresponding to: a) the pendulum-like tension-compression, $b$ ) the pulsation cycles. 
under tension than compression. This is the consequence of damage deactivation effect and total number of cycles to failure is equal to $N=86$. In case of the pulsation tension test (see Fig. 5b) subsequent hysteresis loops are more oblate and exhibit symmetric drop of the force amplitude, leading to characteristic drift of the diagram towards negative magnitudes of the force $P$. Since now the amplitude of displacement $\Delta l$ is equal to one half of the previous one, corresponding total number of cycles to failure is bigger and equal to $N=427$.

\section{Summary}

Results obtained in numerical modeling of the cylindrical shell made of Al-2024 fully confirm that neither geometrical nor material imperfections are necessary to initiate the neck effect. Both examples presented in this work, comprising the cylindrical shell working: in elastic range or in elastic-plastic range including damage, are solved by the use of three independent numerical routines: the finite difference scheme, a direct integration with the fourth-order Runge-Kutta technique and the FEM code. The first example shows location of the neck in very narrow zone between the gauge length and the adjacent conical profile. Nevertheless, combined effects of plastic hardening and the damage, cause the motion of neck which finally localizes in the middle of gauge length.

\section{Acknowledgments}

The authors gratefully acknowledge the support rendered by the Institute of Applied Informatics and the Institute of Applied Mechanics, Cracow University of Technology.

\section{References}

[1] P.W. Bridgman, The stress distribution at the neck of a tension specimen, Trans. ASME, 32 (1944) 533-574.

[2] C.R. Cowper, E.T. Onat, The initiation of necking and buckling in plane plastic flow, Proc. 4th U.S. Nat. Congr. Appl. Mech. (1962) 1023-1029.

[3] N.N. Davidenkov, N.E. Spiridonova, Analysis of stress state in the neck of tensile specimen, Zavodska Laboratoria, Vol. XI, No. 6 (1945) (in Russian).

[4] A. Ganczarski, J. Skrzypek, Plasticity of engineering materials, Cracow University of Technology Publ. House, Kraków, 2007 (in Polish).

[5] J. Lemaite, J.-L. Chaboche, Méchanique des matériaux solides, Bordas, Paris, 1985.

[6] N.N. Malinin, J. Rżysko, Mechanics of materials, PWN, Warsaw, 1981 (in Polish).

[7] D.R.J. Owen, E. Hinton, Finite elements in plasticity: Theory and Practice, Pineridge Press Ltd., Swansea, 1980.

[8] M. Cegielski, Effect of continuous damage deactivation in CDM, PhD Thesis of Cracow University of Technology, 2011 (in Polish).

[9] A. Ganczarski, M. Cegielski, Continuous damage deactivation in modeling of cycle fatigue of engineering materials, Procedia Eng. 2(1) (2010) 1057-1066.

https://doi.org/10.1016/j.proeng.2010.03.114

[10] W. Press W., S. Teukolsky, W. Vettering, B. Flannery, Numerical recipes in Fortran, Cambridge Press, Cambridge, 1993.

[11] L. Dietrich, Consideration of material hardening in analysis of combined plastic flow processes, Issues of IPPT PAN, No 53, 1977 (in Polish). 